\title{
STRONG MULTIPLICITY ONE FOR THE SELBERG CLASS
}

\author{
MICHAEL FARMER
}

\begin{abstract}
We study the problem of determining elements of the Selberg class by information on the coefficents of the Dirichlet series at the squares of primes, or information about the zeroes of the functions.
\end{abstract}

\section{INTRODUCTION}

1.1. The Selberg Class. In 5], Selberg axiomatised the expected properties of $L$ functions and introduced the "Selberg class". This is expected to coincide with the class of all arithmetically interesting $L$ functions.

Definition 1.1. The Selberg class is the set of functions $F$ satisfying the following axioms:

- In the half plane $\Re(s)>1$, the function $F(s)$ is given by a Dirichlet series $\sum_{m=1}^{\infty} a_{F}(m) m^{-s}$, where $a_{F}(1)=1$ and $a_{F}(m) \ll_{\varepsilon} m^{\varepsilon}$ for any $\varepsilon>0$. The estimate $a_{F}(m) \ll_{\varepsilon} m^{\varepsilon}$ is known as the Ramunujan hypothesis.

- There exists a natural number $m_{F}$ such that $(s-1)^{m_{F}} F(s)$ extends to an analytic function of finite order in the entire complex plane.

- There exists $Q_{F}>0, \lambda_{j}(F)>0, \mu_{j}(F), w_{F} \in \mathbb{C}$ with $\Re\left(\mu_{j}(F)\right) \geq 0$ and $\left|w_{F}\right|=1$, such that the function $\Phi_{F}(s)=Q_{F}^{s} \Gamma_{F}(s) F(s)$, where

$$
\Gamma_{F}(s)=\prod_{j=1}^{N_{F}} \Gamma\left(\lambda_{j}(F) s+\mu_{j}(F)\right),
$$

satisfies the functional equation $\Phi_{F}(s)=w_{F} \overline{\Phi_{F}}(1-s)$. Here we use the notation that for any function $f, \bar{f}(s)=\overline{f(\bar{s})}$. Recall that the degree of $F$ is defined to be the $\sum_{j=1}^{N_{f}} 2 \lambda_{j}(F)$.

- $F$ satisfies an Euler product formula. We can express $\log (F(s))$ as a Dirichlet series

$$
\log (F(s))=\sum_{m=2}^{\infty} \frac{b_{F}(m) \Lambda(m)}{m^{s} \log m}
$$

where $b_{F}(m) \ll m^{\theta}$ for some $\theta<1 / 2$. We adopt the convention that $b_{F}(m)$ is defined to be zero when $m$ is not a prime power.

1.2. Previous results. It is generally believed that the Selberg class satisfies the "strong multiplicity one" principle. This states that if $F, G$ are two elements of the Selberg class with $a_{F}(p)=a_{G}(p)$ (equivalently $b_{F}(p)=b_{G}(p)$ ) for all but finitely many primes $p$, then $F=G$. This result is not known in this generality and historically more assumptions have been made to establish similar results. In [1, Kaczorowski and Perelli established this under an assumption on the coefficients at the squares of primes, namely that $\left|a_{F}\left(p^{2}\right)-a_{G}\left(p^{2}\right)\right|$ is bounded on average, so for all large $x$,

$$
\sum_{p \leq e^{x}} \frac{\left|a_{F}\left(p^{2}\right)-a_{G}\left(p^{2}\right)\right|^{2}}{p} \log p \ll x .
$$

In 6], Soundararajan improved on this result by weakening the assumption to

$$
\sum_{p \leq e^{x}} \frac{\left|a_{F}\left(p^{2}\right)-a_{G}\left(p^{2}\right)\right|^{2}}{p} \log p \ll \exp \left(\frac{x}{\log x\left(\log _{2} x\right)^{5}}\right),
$$

where we adopt the convention that $\log _{2} x=\log \log x$. In this paper, we weaken this assumption again by replacing the $\left(\log _{2} x\right)^{-5}$ term by $\varepsilon$. We also establish a similar result assuming no information on the coefficients at squares of primes, but instead use information on the zeroes of $F$ and $G$. One could prove a more general result interpolating between the two cases, but we do not do this. 
1.3. Statement of Results. Write the (non-trivial) zeroes of $F, \rho_{F}$, as $1 / 2+i \gamma_{F}$, where $\gamma_{F} \in \mathbb{C}$. Let $Z_{F}(T)$ denote the multiset of $\gamma_{F} \in \mathbb{R}$, with $\left|\gamma_{F}\right| \leq T$. Recall the symmetric difference notation for (multi)sets $A, B$,

$$
A \triangle B=(A \backslash B) \cup(B \backslash A) .
$$

Theorem 1.1. Suppose $F$ and $G$ are elements of the Selberg class with $a_{F}(p)=a_{G}(p)$ (equivalently $\left.b_{F}(p)=b_{G}(p)\right)$ for all $p \notin E$, where $E$ is a thin set of primes in the sense that

$$
\#\{p \in E: p \leq x\} \ll x^{1 / 2-\delta}
$$

for some fixed $\delta>0$. Suppose either

or

$$
\left|Z_{F}(T) \triangle Z_{G}(T)\right|=O\left(T \frac{\log T}{\log _{2} T}\right),
$$

$$
\sum_{p \leq e^{x}} \frac{\left|a_{F}\left(p^{2}\right)-a_{G}\left(p^{2}\right)\right|^{2}}{p} \log p \ll_{\varepsilon} \exp \left(\frac{\varepsilon x}{\log x}\right), \text { for all } \varepsilon>0 \text {. }
$$

Then $F=G$.

Our method of proof is similar to that of 6 , but we can improve on the results by not choosing a smooth function in the explicit formula, but allowing the smoothness to be a parameter we can also control. This extra degree of freedom allows us to improve the result, but falls short of the conjectured result.

The proof of both cases will be similar, but to make it more concise we shall define the following function.

$$
\rho(T)= \begin{cases}\frac{\log T}{\log _{2} T}, & \text { if (i) holds } \\ \log T, & \text { otherwise. }\end{cases}
$$

This will arise in the proof from the density of the zeroes of $F, G$.

\section{PRELIMINARIES}

We shall need the following unconditional estimate which only relies on the Ramunujan hypothesis (first axiom for Selberg class). For any $\varepsilon>0$, we have

$$
\sum_{p \leq e^{x}} \frac{\left|a_{F}\left(p^{2}\right)-a_{G}\left(p^{2}\right)\right|^{2}}{p} \log p \ll_{\varepsilon} e^{\varepsilon x} .
$$

Let

$$
A_{\varepsilon}(x)= \begin{cases}\exp \left(\frac{\varepsilon x}{\log x}\right), & \text { if (ii) holds } \\ \exp (\varepsilon x), & \text { otherwise. }\end{cases}
$$

We will show the that (non-trivial) zeroes and poles of $F$ and $G$ coincide except on the critical line $\Re(s)=1 / 2$ when $F$ and $G$ satisfy (1). Write $c(n)=b_{F}(n)-b_{G}(n)$ and consider the function $-F^{\prime} / F(s)+G^{\prime} / G(s)$. For $\Re(s)>3 / 2$, the Euler product implies $F$ and $G$ have no zeroes, so the function is well defined in this region. For $\Re(s)>3 / 2$,

$$
-\frac{F^{\prime}}{F}(s)+\frac{G^{\prime}}{G}(s)=\sum_{m=1}^{\infty} \frac{c(m) \Lambda(m)}{m^{s}}=\sum_{k=1}^{\infty} \sum_{p} \frac{c\left(p^{k}\right) \log (p)}{p^{k s}} .
$$

For $k=1, c(p)=0$ unless $p \in E$. Fix arbitrary $\varepsilon>0$. Then for all $s$ with $\Re(s)>1 / 2-\delta+2 \varepsilon$ we have $\Re(s)-\varepsilon>1 / 2-\delta+\varepsilon$. Using $c(p), \log p \ll_{\varepsilon} p^{\varepsilon}$ implies

$$
\sum_{p \leq x} \frac{c(p) \log p}{p^{s}} \ll \sum_{p \leq x} \frac{\mathbb{1}_{E}(p)}{p^{\Re(s)-\varepsilon}} .
$$

Summing by parts and using (1), the RHS converges uniformly as $x$ goes to infinity . Hence $\sum_{p} c(p) \log p / p^{s}$ is analytic for $\Re(s)>1 / 2-\delta$. Recall $c(m) \ll m^{\theta}$ for some fixed $\theta<1 / 2$. Fix an integer $M$ such that $M(1 / 2-\theta)>1$. Then

$$
\sum_{k=2}^{\infty} \sum_{p} \frac{c\left(p^{k}\right) \log p}{p^{k s}}=\sum_{p}\left(\sum_{k=2}^{M-1} \frac{c\left(p^{k}\right) \log p}{p^{k s}}+\sum_{k=M}^{\infty} \frac{c\left(p^{k}\right) \log p}{p^{k s}}\right)
$$


The first term is analytic for $\Re(s)>1 / 2$ since $c\left(p^{k}\right) \ll_{\varepsilon} p^{\varepsilon}$ for $2 \leq k \leq M-1$. For $\Re(s)>1 / 2$,

$$
\sum_{p} \sum_{k=M}^{\infty} \frac{c\left(p^{k}\right) \log p}{p^{k s}} \ll \sum_{p} \sum_{k=M}^{\infty} \frac{\log p}{p^{k(\Re(s)-\theta)}} \ll \sum_{p} \frac{\log p}{p^{M(\Re(s)-\theta)}} \leq \sum_{p} \frac{\log p}{p^{M\left(\frac{1}{2}-\theta\right)}}=O(1)
$$

so $\sum_{k=4}^{\infty} \sum_{p} c\left(p^{k}\right) \log p / p^{k s}$ is analytic for $\Re(s)>1 / 2$. Putting this all together, the RHS of 37 is analytic for $\Re(s)>1 / 2$ which allows us to analytically continue the LHS to $\Re(s)>1 / 2$. It follows that the zeroes and poles of $F$ and $G$ in this region agree (including multiplicities). Repeating the argument for $\bar{F}(s)=\sum_{n=1}^{\infty} \overline{a_{n}} n^{-s}$ and $\bar{G}(s)$ shows that their poles and zeroes also coincide for $\Re(s)>1 / 2$. Putting this together with the functional equation shows that $\Phi_{F}$ and $\Phi_{G}$ have the same zeroes except for possibly on $\Re(s)=1 / 2$ and poles of the same multiplicity at $s=1$.

Let $T$ be a parameter which shall be taken to infinity. A standard application of the argument principle implies that the (non-trivial) zeroes of $F, \rho_{F}$, satisfy the following estimate

$$
\#\left\{\rho_{F}:\left|\Im\left(\rho_{F}\right)\right| \leq T\right\}=\frac{d_{F}}{\pi} T \log T+C_{F} T+O_{F}(\log T)
$$

where $C_{F}$ is a constant and $d_{F}$ is the degree of $F$.

Let $\mu \geq 3$ be a real parameter to be chosen later (it will be chosen so $\mu \asymp \log \rho(T)$ ). For $x \in \mathbb{R}$, define

$$
g_{\mu}(x)=\frac{2^{2 \mu}}{\pi \sqrt{\mu}\left(\begin{array}{c}
2 \mu \\
\mu
\end{array}\right)} \begin{cases}\left(1-x^{2}\right)^{\mu-1 / 2}, & \text { if }|x| \leq 1 \\
0, & \text { otherwise }\end{cases}
$$

The function $g_{\mu}$ has Fourier transform

$$
h_{\mu}(t)=\frac{\Gamma(\mu+1)}{\sqrt{\mu}} J_{\mu}(|t|)\left(\frac{2}{|t|}\right)^{\mu}
$$

where $J_{\mu}(t)$ is the Bessel function of the first kind. This is a solution to the Bessel differential equation

$$
t^{2} \frac{d^{2} y}{d t^{2}}+t \frac{d y}{d t}+\left(t^{2}-\mu^{2}\right) y=0
$$

By 2 we have $J_{\mu}(|t|)=O(1)$ uniformly for $\mu \geq 3, t \in \mathbb{R}$. Let us write $C_{\mu}=\Gamma(\mu+1) 2^{\mu} / \sqrt{\mu}$. We call the estimate

$$
\left|h_{\mu}(t)\right| \leq C_{\mu}\left(\frac{1}{|t|}\right)^{\mu}
$$

the asymptotic estimate for $h_{\mu}$.

An important property of $g_{\mu}$ we shall use repeatedly throughout the paper is the following bound. By Stirling's formula, for any $x \in \mathbb{R}$,

$$
g_{\mu}(x) \leq g_{\mu}(0) \asymp 1 .
$$

Also, we can recover $g_{\mu}$ from $h_{\mu}$ by means of the Fourier inversion formula

$$
g_{\mu}(x)=\frac{1}{2 \pi} \int_{-\infty}^{\infty} h_{\mu}(t) e^{-i x t} d t .
$$

Consider a $C^{2}$ function $u$ which is compactly supported with Fourier transform $v$. Recall we write the (non-trivial) zeroes of $F, \rho_{F}$ as $1 / 2+i \gamma_{F}$. Approximating $C^{2}$ functions by smooth functions we can use 44, Proposition 2.1] to get the following explicit formula which relates the (non-trivial) zeroes of $F$ to $b_{F}(m) \Lambda(m)$ at prime powers.

$$
\begin{aligned}
\sum_{\gamma_{F}} v\left(\gamma_{F}\right) & =m_{f}\left(v\left(\frac{-i}{2}\right)+v\left(\frac{i}{2}\right)\right) \\
& +\frac{1}{2 \pi} \int_{-\infty}^{\infty} v(r)\left(2 \log Q_{F}+\frac{\Gamma_{F}^{\prime}}{\Gamma_{F}}\left(\frac{1}{2}+i r\right)+\frac{\overline{\Gamma_{F}^{\prime}}}{\overline{\Gamma_{F}}}\left(\frac{1}{2}-i r\right)\right) d r \\
& -\sum_{m=1}^{\infty}\left(\frac{b_{F}(m) \Lambda(m)}{\sqrt{m}} u(\log m)+\frac{\overline{b_{F}(m)} \Lambda(m)}{\sqrt{m}} u(-\log m)\right),
\end{aligned}
$$

where we sum over non-trivial zeroes of $F$ with multiplicity.

Let $t \in[T, 2 T]$. Let $L$ be another parameter depending on $T$ (which will chosen so $L \asymp \rho(T) \log \rho(T)$. 
Notice that $g_{\mu}$ is not a smooth function, but for $\mu \geq 3, g_{\mu}$ is $C^{2}$. Hence by considering $v(r)=h_{\mu}(L(r-t))$ and using the Fourier inversion formula we deduce

$$
\begin{aligned}
\left.\sum_{\gamma_{F}} h_{\mu}\left(L\left(\gamma_{f}-t\right)\right)\right) & =m_{f}\left(h_{\mu}\left(L\left(\frac{-i}{2}-t\right)\right)+h_{\mu}\left(L\left(\frac{i}{2}+t\right)\right)\right) \\
& +\frac{1}{2 \pi} \int_{-\infty}^{\infty} h_{\mu}(L(r-t))\left(2 \log Q_{F}+\frac{\Gamma_{F}^{\prime}}{\Gamma_{F}}\left(\frac{1}{2}+i r\right)+\frac{\overline{\Gamma_{F}^{\prime}}}{\overline{\Gamma_{F}}}\left(\frac{1}{2}-i r\right)\right) d r \\
& -\frac{1}{L} \sum_{m=1}^{\infty}\left(\frac{b_{F}(m) \Lambda(m)}{m^{1 / 2+i t}} g_{\mu}\left(\frac{\log m}{L}\right)+\frac{\overline{b_{F}(m)} \Lambda(m)}{m^{1 / 2-i t}} g_{\mu}\left(\frac{-\log m}{L}\right)\right) .
\end{aligned}
$$

Denote the middle and third terms by $H_{F}(t, L, \mu), D_{F}(t, L . \mu)$ respectively. We shall use the following lemma to be proven at the end.

Lemma 2.1. For our choice of $\mu$ and $L$,

$$
H_{F}(t, L, \mu)=g_{\mu}(0) \frac{d_{F} \log T}{L}+\frac{O\left(E_{\mu}(T)\right)}{L}
$$

where

$$
E_{\mu}(T)=\frac{C_{\mu}}{T}+1 \quad \text { and } \quad C_{\mu}=\Gamma(\mu+1) 2^{\mu} / \sqrt{\mu}
$$

\section{PROOF OF THEOREM}

By our observations on the zeroes of $F, G$ above, we have

$$
Z_{F}(t, L, \mu)-Z_{G}(t, L, \mu)=H_{F}(t, L, \mu)-H_{G}(t, L, \mu)-D_{F}(t, L, \mu)+D_{G}(t, L, \mu)
$$

where

$$
\left.Z_{F}(t, L, \mu)=\sum_{\gamma_{F} \in \mathbb{R}} h_{\mu}\left(L\left(\gamma_{f}-t\right)\right)\right) .
$$

We now want to record an estimate for $D_{F}(t, L, \mu)-D_{G}(t, L, \mu)$. Choose $\varepsilon>0$ such that $1 / 2-\varepsilon>1 / 2-\delta$, where $\delta$ is from (1). Since $c(p) \ll_{\varepsilon} p^{\varepsilon}$ and using (6), (for the rest of the paper we shall do this without mention), we have

$$
\sum_{p} \frac{|c(p)| g_{\mu}(\log p / L) \log p}{\sqrt{p}} \ll \sum_{p \in E} \frac{1}{p^{1 / 2-\varepsilon}} \ll 1
$$

Since $c(m) \ll m^{\theta}$ we see that

$$
\sum_{k \geq 3} \sum_{p} \frac{\left|c\left(p^{k}\right)\right| g_{\mu}(k \log p / L) \log p}{p^{k / 2}} \ll 1 .
$$

By considering the logarithmic derivative of $F$, it is easy to see show that $b_{F}\left(p^{2}\right)=a_{F}\left(p^{2}\right)-a_{F}(p) b_{F}(p) / 2$. Noticing that again by (1)

$$
\sum_{p} \frac{\left|a_{F}(p) b_{F}(p)-a_{G}(p) b_{G}(p)\right| g_{\mu}(2 \log p / L) \log p}{p} \ll 1,
$$

we have that

$$
\begin{aligned}
L\left(D_{F}(t, L, \mu)-D_{G}(t, L, \mu)\right) \ll 1 & +\left|\sum_{p} \frac{a_{F}\left(p^{2}\right)-a_{G}\left(p^{2}\right)}{p^{1+2 i t}} g_{\mu}\left(\frac{2 \log p}{L}\right) \log p\right| \\
& +\left|\sum_{p} \frac{\overline{a_{F}\left(p^{2}\right)}-\overline{a_{G}\left(p^{2}\right)}}{p^{1-2 i t}} g_{\mu}\left(\frac{-2 \log p}{L}\right) \log p\right| .
\end{aligned}
$$


Using a mean-value estimate from Montgomery and Vaughan [3, Corollary 3], and using the fact $g_{\mu}$ is compactly supported on $[-1,1]$, we have that

$$
\begin{aligned}
\int_{T}^{2 T}\left|\sum_{p} \frac{a_{F}\left(p^{2}\right)-a_{G}\left(p^{2}\right)}{p^{1+2 i t}} g_{\mu}\left(\frac{2 \log p}{L}\right) \log p\right|^{2} d t & \ll \sum_{p \leq e^{L / 2}} \frac{\left|a_{F}\left(p^{2}\right)-a_{G}\left(p^{2}\right)\right|^{2}}{p^{2}}(T+p) \log ^{2} p \\
& \ll T+\sum_{p \leq e^{L / 2}} \frac{\left|a_{F}\left(p^{2}\right)-a_{G}\left(p^{2}\right)\right|^{2}}{p} \log ^{2} p \\
& \ll T+O_{\epsilon}\left(\frac{L}{2} A_{\varepsilon}(L / 2)\right),
\end{aligned}
$$

where the last line comes from (2). Dealing with the cross terms using Cauchy's inequality, we deduce that

$$
\int_{T}^{2 T}\left(L\left(D_{F}(t, L, \mu)-D_{G}(t, L, \mu)\right)\right)^{2} d t \ll T+O_{\varepsilon}\left(A_{\varepsilon}(L)\right) .
$$

Let $W \geq 1$ be a large constant to be chosen later. Let $\mathcal{L}=\mathcal{L}(W)$ denote the set of $t \in[T, 2 T]$ such that there exists $\gamma \in Z_{F}(\infty) \triangle Z_{G}(\infty)$ in $\left(t-\frac{1}{W \rho(T)}, t+\frac{1}{W \rho(T)}\right)$. Notice in either case (i) or (ii), (i) or 4 implies for large enough $T, \operatorname{Meas}(\mathcal{L}) \ll T \rho(2 T+1) / W \rho(T) \ll T / W$. Let $\widetilde{\mathcal{L}}$ denote $[T, 2 T] \backslash \mathcal{L}$. Then

$$
\begin{aligned}
& L \int_{t \in \tilde{\mathcal{L}}}\left|Z_{F}(t, L, \mu)-Z_{G}(t, L, \mu)\right| d t \\
= & L \int_{T}^{2 T}\left|\sum_{\substack{\gamma_{F} \in \mathbb{R} \\
\left|\gamma_{F}-t\right| \geq 1 / W \rho(T)}} h_{\mu}\left(L\left(\gamma_{F}-t\right)\right)-\sum_{\substack{\gamma_{G} \in \mathbb{R} \\
\left|\gamma_{G}-t\right| \geq 1 / W \rho(T)}} h_{\mu}\left(L\left(\gamma_{G}-t\right)\right)\right| d t \\
= & L \int_{T}^{2 T}\left|\sum_{\substack{|\gamma-t| \geq 1 / W \rho(T) \\
\gamma \in Z_{F}(\infty) \Delta Z_{G}(\infty)}} \pm h_{\mu}(L(\gamma-t))\right| d t .
\end{aligned}
$$

We deal now with the contribution of zeroes outside $(T-1,2 T+1)$. If the distance of $\gamma$ from $[T, 2 T]$ exceeds $m$ then the contribution from any term on the RHS of the above equation is $\leq \mu^{-1 / 2} \Gamma(\mu+1)(2 / L m)^{\mu}$ by (5). Since there are $\ll \log (T(m+1))$ ordinates $\gamma$ whose distance is between $m$ and $m+1$ by (4), the total contribution is $\ll \mu^{-1 / 2} \Gamma(\mu+1)(2 / L)^{\mu} \log (T)$. This is because $\sum_{m=1}^{\infty} m^{-\mu} \log (m+1)$ and $\sum_{m=1}^{\infty} m^{-\mu}$ are $O(1)$ independent of $\mu \geq 3$. This means

$$
\begin{aligned}
& L \int_{t \in \tilde{\mathcal{L}}}\left|Z_{F}(t, L, \mu)-Z_{G}(t, L, \mu)\right| d t \\
\ll & \mu^{-1 / 2} \Gamma(\mu+1)(2 / L)^{\mu} \log (T)+\sum_{\substack{\gamma \in(T-1,2 T+1) \\
\gamma \in Z_{F}(2 T+1) \triangle Z_{G}(2 T+1)}} \int_{|y| \geq L / W \rho(T)}\left|h_{\mu}(y)\right| d y \\
\ll & \mu^{-1 / 2} \Gamma(\mu+1)(2 / L)^{\mu} \log (T)+T \rho(T) \int_{|y| \geq L / W \rho(T)}\left|h_{\mu}(y)\right| d y,
\end{aligned}
$$

where the last line comes from (4).

Suppose now that $F \neq G$, so there is an integer $m$ such that $c(m) \neq 0$. Using $(12)$ we see that

$$
\begin{aligned}
& L \int_{t \in \tilde{\mathcal{L}}} m^{i t}\left(Z_{F}(t, L, \mu)-Z_{G}(t, L, \mu)\right) d t \\
\ll & \mu^{-1 / 2} \Gamma(\mu+1)(2 / L)^{\mu} \log (T)+T \rho(T) \int_{|y| \geq L / W \rho(T)}\left|h_{\mu}(y)\right| d y .
\end{aligned}
$$

However using (10) this is also equal to

$$
L \int_{t \in \tilde{\mathcal{L}}} m^{i t}\left(H_{F}(t, L, \mu)-H_{G}(t, L, \mu)-D_{F}(t, L, \mu)+D_{G}(t, L, \mu)\right) d t .
$$


From [6], (1) tells us that $d_{F}=d_{G}$. Recall that for large $T, \operatorname{Meas}(\mathcal{L}) \ll T / W$. So for large $T$ we have

$$
L \int_{t \in \mathcal{L}}\left|H_{F}(t, L, \mu)-H_{G}(t, L, \mu)\right| d t \ll E_{\mu}(T) \operatorname{Meas}(\mathcal{L}) \ll E_{\mu}(T) \frac{T}{W} .
$$

We will also need the following lemma proved at the end of the paper.

Lemma 3.1. Let F, $G$ lie in the Selberg class and satisfy (1). For our choice of $\mu, L$, we have the following estimate,

$$
\int_{T}^{2 T} L m^{i t}\left(H_{F}(t, L, \mu)-H_{G}(t, L, \mu)\right) d t \ll T\left(\mu^{-1 / 4}+\frac{C_{\mu}}{T}\right)+E_{\mu}(2 T)+E_{\mu}(T) .
$$

Putting our above formula together with the lemma we have

$$
L \int_{t \in \tilde{\mathcal{L}}} m^{i t}\left(H_{F}(t, L, \mu)-H_{G}(t, L, \mu)\right) d t \ll T\left(\mu^{-1 / 4}+\frac{C_{\mu}}{T}\right)+E_{\mu}(2 T)+E_{\mu}(T) \frac{T}{W} .
$$

By Cauchy's inequality and (11) we have that

Also integrating term by term we see that

$$
\int_{t \in \mathcal{L}} L\left|D_{F}(t, L, \mu)-D_{G}(t, L, \mu)\right| d t \ll \frac{T+O_{\varepsilon}\left(A_{\varepsilon}(L)\right)}{\sqrt{W}} .
$$

$$
\int_{T}^{2 T} L m^{i t}\left(D_{F}(t, L, \mu)-D_{G}(t, L, \mu)\right) d t=T \frac{c(m)}{\sqrt{m}} \Lambda(m) g_{\mu}\left(\frac{\log m}{L}\right)+O\left(\sum_{u \leq e^{L}} \frac{|c(u)| \log u}{\sqrt{u}}\right) .
$$

By similar calculations as done earlier in section 2 we can check that

$$
\sum_{u \leq e^{L}} \frac{|c(u)| \log u}{\sqrt{u}}=O_{\varepsilon}\left(A_{\varepsilon}(L)\right)
$$

We conclude that

$$
\int_{t \in \tilde{\mathcal{L}}} L m^{i t}\left(D_{F}(t, L, \mu)-D_{G}(t, L, \mu)\right) d t=T \frac{c(m)}{\sqrt{m}} \Lambda(m) g_{\mu}\left(\frac{\log m}{L}\right)+O\left(\frac{T}{\sqrt{W}}\right)+O_{\varepsilon}\left(A_{\varepsilon}(L)\right) .
$$

Since $c(m)=0$ except on prime powers, we have that $\Lambda(m) \neq 0$. Putting all information we have collected together ( (13), 14), 115), 16) $)$,

$$
\begin{aligned}
1 & \ll \frac{c(m)}{\sqrt{m}} \Lambda(m)=\frac{\int_{t \in \tilde{\mathcal{L}}} L m^{i t}\left(D_{F}(t, L, \mu)-D_{G}(t, L, \mu)\right) d t+O\left(\frac{T}{\sqrt{W}}\right)+O_{\epsilon}\left(A_{\epsilon}(L)\right)}{T g_{\mu}\left(\frac{\log m}{L}\right)} \\
& \ll \frac{1}{T g_{\mu}(\log m / L)}\left(T\left(\mu^{-1 / 4}+C_{\mu} / T\right)+E_{\mu}(2 T)+E_{\mu}(T) \frac{T}{W}+\mu^{-1 / 2} \Gamma(\mu+1)(2 / L)^{\mu} \log (T)\right. \\
& \left.+T \rho(T) \int_{|y| \geq L / W \rho(T)}\left|h_{\mu}(y)\right| d y+\frac{T}{\sqrt{W}}+O_{\varepsilon}\left(A_{\varepsilon}(L)\right)\right) .
\end{aligned}
$$

By Stirling's formula we have $g_{\mu}(\log m / L) \sim\left(1-(\log m / L)^{2}\right)^{\mu-1 / 2}$. Using the identity $1-x^{2} \geq e^{-2 x^{2}}$ for $|x|<1 / 2$, together with the fact $L$ goes to infinity with $T$, for large enough $T$ we have that $e^{-2(\mu-1 / 2)(\log m / L)^{2}} \ll g_{\mu}(\log m / L)$.

We shall get a contradiction in (17) to complete the proof as then $c(m)=0$ for all $m$. To get a contradiction we shall make the following choices for $L, \mu, W, \varepsilon$. Let $W$ be a sufficiently large constant to be chosen in a moment and let $\varepsilon=1 / 2 W^{2}$. Finally, let $\mu=L / 2 W \rho(T)$ and $L=W^{2} \rho(T) \log (\rho(T))$. Under these choices, for large enough $T, 1 \ll g_{\mu}(\log m / L)$.

For the integral term,

$$
\int_{|y| \geq L / W \rho(T)}\left|h_{\mu}(y)\right| d y \leq \frac{\Gamma(\mu+1)}{\sqrt{\mu}} \int_{|y| \geq L / W \rho(T)}\left(\frac{2}{|y|}\right)^{\mu} d y \ll \frac{1}{\sqrt{\mu}} \frac{\Gamma(\mu+1)}{\mu-1}\left(\frac{2}{L / W \rho(T)}\right)^{\mu-1} .
$$

Since $\mu=L / 2 W \rho(T)$, using Stirling's formula, we obtain

$$
\int_{|y| \geq L / W \rho(T)}\left|h_{\mu}(y)\right| d y \ll e^{-\mu} .
$$


This estimate shows that $\rho(T) \int_{|y| \geq L / W \rho(T)}\left|h_{\mu}(y)\right| d y \rightarrow 0$ as $T \rightarrow \infty$ as desired.

For the contribution of zeroes outside $(T-1,2 T+1)$, using the formula for $\mu$ in terms of $L$ and Stirling's formula, we get

$$
\begin{aligned}
\frac{\mu^{-1 / 2} \Gamma(\mu+1)(2 / L)^{\mu} \log (T)}{T} & =\frac{\Gamma(\mu+1)(1 / \mu W \rho(T))^{\mu} \log (T)}{\sqrt{\mu} T} \\
& \ll \frac{e^{-\mu} \log (T)}{(W \rho(T))^{\mu} T} .
\end{aligned}
$$

This converges to 0 as $T \rightarrow \infty$ as desired.

For the final term, under the choice of $\varepsilon, A_{\epsilon}(L) / T \rightarrow 0$ as $T \rightarrow \infty$.

By Stirling's formula,

$$
C_{\mu} \ll\left(\frac{2}{e}\right)^{\mu} \mu^{\mu}
$$

Since $\mu^{\mu} / T \rightarrow 0$ as $T \rightarrow \infty$, we deduce that $C_{\mu} / T \rightarrow 0$ as $T \rightarrow \infty$. Hence $E_{\mu}(T)$ converges to 1 as $T \rightarrow \infty$. Putting this all together, taking $T$ to be sufficiently large 17 implies that

$$
1 \ll \frac{1}{\sqrt{W}},
$$

which is clearly contradicted by choosing sufficiently large $W$, completing the proof.

\section{Proofs of Lemmas}

Recall our definitions of $C_{\mu}, E_{\mu}(T)$ from Lemma 2.1. Also recall that $t \in[T, 2 T], \mu \asymp \log \rho(T)$, $L \asymp \rho(T) \log \rho(T)$. We shall remove any $F$ or $G$ subscripts to make it more concise.

Proof of Lemma 2.1. Looking at the integral formula for $H(t, L, \mu)$. For the constant term in the integral, by the Fourier inversion formula

$$
\int_{-\infty}^{\infty} h_{\mu}(L(r-t)) d r=\frac{1}{L} g_{\mu}(0)
$$

Denote $\left|\lambda_{j}\left(\frac{1}{2}+i r\right)+\mu_{j}\right|$ as $l_{j}(r)$. Since $l_{j}(r)$ is uniformly bounded away from 0 for $r \in \mathbb{R}$ (by assumptions on $\lambda_{j}, \mu_{j}$ ), Stirling's formula implies the digamma term is equal to

$$
\begin{aligned}
& \sum_{j=1}^{N} 2 \lambda_{j} \int_{-\infty}^{\infty} h_{\mu}(L(r-t)) \Re \log \left(\lambda_{j}\left(\frac{1}{2}+i r\right)+\mu_{j}\right) d r+\sum_{j=1}^{N} \int_{-\infty}^{\infty} O\left(\frac{1}{l_{j}(r)}\right)\left|h_{\mu}(L(r-t))\right| d r \\
& =\sum_{j=1}^{N} 2 \lambda_{j} \int_{-\infty}^{\infty} h_{\mu}(L(r-t)) \log \left(l_{j}(r)\right) d r+\sum_{j=1}^{N} \int_{-\infty}^{\infty} O\left(\frac{1}{l_{j}(r)}\right)\left|h_{\mu}(L(r-t))\right| d r .
\end{aligned}
$$

For the error term, using the trivial estimate $\left|h_{\mu}(u)\right| \leq h_{\mu}(0)=1 / \sqrt{\mu}$, and splitting the integral up into suitable regions, we have

$$
\int_{-\infty}^{\infty} \frac{1}{l_{j}(r)}\left|h_{\mu}(L(r-t))\right| d r \ll \frac{1}{L}\left(\frac{1}{\mu^{1 / 4}}+\int_{|u|>\mu^{1 / 4}} \frac{1}{l_{j}\left(\frac{u}{L}+t\right)}\left|h_{\mu}(u)\right| d u\right) .
$$

When $u>\mu^{1 / 4}, 1 / l_{j}\left(\frac{u}{L}+t\right) \ll 1 /\left(\frac{u}{L}+t\right) \leq 1 / t \leq 1 / T$, so

$$
\int_{u>\mu^{1 / 4}} \frac{1}{l_{j}\left(\frac{u}{L}+t\right)}\left|h_{\mu}(u)\right| d u \ll \frac{C_{\mu}}{T\left(\mu^{1 / 4}\right)^{\mu-1}(\mu-1)} \ll \frac{C_{\mu}}{T} .
$$

We still need to consider the integral when $u \leq-\mu^{1 / 4}$. We would like to use the estimate $1 / l_{j}\left(\frac{u}{L}+t\right) \ll 1 /\left|\frac{u}{L}+t\right|$, however it is not useful in a neighbourhood of $-t L$ (which definitely lies in this region by our choice of $\mu, L)$. In this region we use $1 / l_{j}\left(\frac{u}{L}+t\right)=O(1)$. Let $a=L T / 2$. We have the estimate

$$
\int_{-t L-a}^{-t L+a} \frac{1}{l_{j}\left(\frac{u}{L}+t\right)}\left|h_{\mu}(u)\right| d u \ll \frac{C_{\mu}}{(\mu-1)(L t-a)^{\mu-1}} \ll \frac{C_{\mu}}{(\mu-1) a^{\mu-1}} \ll \frac{C_{\mu}}{T} .
$$

On $\left(-L t+a,-\mu^{1 / 4}\right)$, we have $1 /\left|\frac{u}{L}+t\right| \ll 1 / T$, so

$$
\int_{-t L+a}^{-\mu^{1 / 4}} \frac{1}{l_{j}\left(\frac{u}{L}+t\right)}\left|h_{\mu}(u)\right| d u \ll \frac{C_{\mu}}{T\left(\mu^{1 / 4}\right)^{\mu-1}(\mu-1)} \ll \frac{C_{\mu}}{T} .
$$


Finally, by the same analysis,

$$
\int_{-\infty}^{-t L-a} \frac{1}{l_{j}\left(\frac{u}{L}+t\right)}\left|h_{\mu}(u)\right| d u \ll \frac{C_{\mu}}{T(\mu-1)(t L+a)^{\mu-1} \ll} \ll \frac{C_{\mu}}{T(\mu-1)(3 T L / 2)^{\mu-1}} \ll \frac{C_{\mu}}{T} .
$$

Putting all of our analysis together, we see that

$$
\int_{-\infty}^{\infty} \frac{1}{l_{j}(r)}\left|h_{\mu}(L(r-t))\right| d r \ll \frac{1}{L}\left(\frac{1}{\mu^{1 / 4}}+\frac{C_{\mu}}{T}\right) .
$$

We have dealt with the error term in $(19)$. Now for the first term, notice that $l_{j}(T) \sim \lambda_{j} T$, so when $r \in[T-\sqrt{T}, 2 T+\sqrt{T}]$ we have $\log l_{j}(r)=\log T+O(1)$. Fixing $j$ and recalling $t \in[T, 2 T]$, we have

$$
\begin{aligned}
\int_{-\infty}^{\infty} h_{\mu}(L(r-t)) \log \left(l_{j}(r)\right) d r & =\int_{t-\sqrt{T}}^{t+\sqrt{T}} h_{\mu}(L(r-t))(\log T+O(1)) d r \\
& +\int_{|r-t|>\sqrt{T}} h_{\mu}(L(r-t)) \log l_{j}(r) d r .
\end{aligned}
$$

Notice that when $|u|>L \sqrt{T}$, we have $\log l_{j}\left(\frac{u}{L}+t\right) \ll \log (|u|)$ since $\sqrt{t} \ll u$. Integrating by parts we have

$$
\int_{|u|>L \sqrt{T}} h_{\mu}(u) \log l_{j}\left(\frac{u}{L}+t\right) d u \ll \frac{C_{\mu}}{(\mu-1)(L \sqrt{T})^{\mu-1}}\left(\log (L \sqrt{T})+\frac{1}{\mu-1}\right) .
$$

This implies

$$
\int_{|r-t|>\sqrt{T}} h_{\mu}(L(r-t)) \log l_{j}(r) d r \ll \frac{C_{\mu}}{L T} .
$$

For the error term in the first integral in 21],

This also implies

$$
\int_{t-\sqrt{T}}^{t+\sqrt{T}} h_{\mu}(L(r-t)) O(1) d r \ll \frac{C_{\mu}}{L(\mu-1)(L \sqrt{T})^{\mu-1}} \ll \frac{C_{\mu}}{L T} .
$$

$$
\frac{\log (T)}{2 \pi} \int_{t-\sqrt{T}}^{t+\sqrt{T}} h_{\mu}(L(r-t)) d r=\frac{\log (T)}{L}\left(g_{\mu}(0)+O\left(\frac{C_{\mu}}{(\mu-1)(L \sqrt{T})^{\mu-1}}\right)\right) .
$$

Putting this all together,

$$
\frac{1}{2 \pi} \int_{-\infty}^{\infty} h_{\mu}(L(r-t)) \log \left(l_{j}(r)\right) d r=\frac{\log (T)}{L} g_{\mu}(0)+\frac{1}{L} O\left(\frac{C_{\mu}}{T}\right) .
$$

Using the fact $\left.g_{\mu}(0)=O(1),(18),(19), 20\right)$ and 22 show that

$$
H_{F}(t, L, \mu)=g_{\mu}(0) \frac{d_{F} \log (T)}{L}+\frac{1}{L} O\left(E_{\mu}(T)\right) .
$$

Proof of Lemma 3.1. Integrating by parts and using $d_{F}=d_{G}$, which follows from (1) and [6], Lemma 2.1 implies that

$$
\begin{aligned}
\int_{T}^{2 T} L m^{i t}\left(H_{F}(t, L, \mu)-H_{G}(t, L, \mu)\right) d t & =-\frac{1}{i \log m} \int_{T}^{2 T} L \frac{d}{d t}\left(H_{F}(t, L, \mu)-H_{G}(t, L, \mu)\right) m^{i t} d t \\
& +O\left(E_{\mu}(2 T)+E_{\mu}(T)\right) .
\end{aligned}
$$

Hence to complete the proof we just need to estimate $L \frac{d}{d t}\left(H_{F}(t, L, \mu)-H_{G}(t, L, \mu)\right)$. Differentiating under the integral expression of $H_{F}(t, L, \mu)-H_{G}(t, L, \mu)$ and integrating by parts again, we get

$$
\begin{aligned}
& L \frac{d}{d t}\left(H_{F}(t, L, \mu)\right)= \\
& -\left.\left(2 \log Q_{F}+\frac{\Gamma_{F}^{\prime}}{\Gamma_{F}}\left(\frac{1}{2}+i r\right)+\frac{\overline{\Gamma_{F}^{\prime}}}{\overline{\Gamma_{F}}}\left(\frac{1}{2}-i r\right)\right) L h_{\mu}(L(r-t))\right|_{r=-\infty} ^{\infty} \\
& +i L \int_{-\infty}^{\infty} h_{\mu}(L(r-t))\left(\left(\frac{\Gamma_{F}^{\prime}}{\Gamma_{F}}\right)^{\prime}\left(\frac{1}{2}+i r\right)-\left(\overline{\frac{\Gamma_{F}^{\prime}}{\overline{\Gamma_{F}}}}\right)^{\prime}\left(\frac{1}{2}-i r\right)\right) d r .
\end{aligned}
$$


By Stirling's formula and our asymptotic estimate on $h_{\mu}$, the first term is zero. By Stirling's formula for the trigamma function, the trigamma terms in the integral are $O\left(1 / l_{j}(r)\right)$. Using $(20)$, we have that the integral term is $\ll \mu^{-1 / 4}+C_{\mu} / T$, thus completing our proof.

\section{REFERENCES}

[1] Jerzy Kaczorowski and Alberto Perelli, Strong multiplicity one for the Selberg class, C. R. Acad. Sci. Paris Sér. I Math. 332 (2001), no. 11, 963-968. MR 1838120

[2] I. Krasikov, Uniform bounds for Bessel functions, J. Appl. Anal. 12 (2006), no. 1, 83-91. MR 2243854

[3] H. L. Montgomery and R. C. Vaughan, Hilbert's inequality, J. London Math. Soc. (2) 8 (1974), 73-82. MR 337775

[4] Zeév Rudnick and Peter Sarnak, Zeros of principal L-functions and random matrix theory, vol. 81, 1996, A celebration of John F. Nash, Jr., pp. 269-322. MR 1395406

[5] Atle Selberg, Old and new conjectures and results about a class of Dirichlet series, Proceedings of the Amalfi Conference on Analytic Number Theory (Maiori, 1989), Univ. Salerno, Salerno, 1992, pp. 367-385. MR 1220477

[6] K. Soundararajan, Strong multiplicity one for the Selberg class, Canad. Math. Bull. 47 (2004), no. 3, $468-474$. MR 2073408

School of Mathematics, University of Bristol, Bristol, BS8 1UG, United Kingdom Email address: michaelfarmer868@gmail.com 